ness of this continuing need for an adequate infrastructure to eliminate the unilateral dependence of villages on certain markets proves that this new approach to development through local integrated non-governmental initiatives from the North and the South does not supersede larger governmental projects in general. However, such projects have to be subordinated and closely interwined with the grass roots programs, which do - as the report proves - qualify for large scale expansion in numbers.

No matter how much the NGO's may be criticized in details, the revolution set in motion by the NGO's of the North and the South deserves respect and attention - as long as it is still peaceful.

Of course, the implications of this report are not unlimited. Many of the findings regarding mistakes in the past have previously been subject to surveys. Some are even known to a wider public. Unquestionably, however, the work of the NGO's of the North and the South, their success and the attitude they formed are not generally known. Not all of the present developmental projects of the "First World" give rise to the assumption that they are the result of due consideration of all the controversial aspects which Schneider points out. Only seldom, the values and culture of the barefooted seem to be reflected, even more seldom, their experience seems to be accepted as know-how. Thus, although no new theory is added to those already existing, the reading of Schneider's critical and encouraging report can be highly recommended to everyone who deals with the situation of the Third World and also for an interested public.

Ulf Marzik

\title{
Peter Hamacher
}

Die Maxime audiatur et altera pars im Völkerrecht

Ein Beitrag zum internationalen Verfahrensrecht unter besonderer Berücksichtigung der Rechtsgutachten des (StIGH) IGH

Springer Verlag Wien, New York 1986

Osterreichische Zeitschrift für öffentliches Recht und Völkerrecht, Supplementum 7

In auffälligem Kontrast zu den innerstaatlichen Rechtsordnungen fristet die Maxime "audiatur et altera pars" in der völkerrechtlichen Diskussion von jeher ein Schattendasein, was daran liegen mag, daß hier der Bereich des Prozeßrechts schon immer eher schwach ausgeprägt war.

In der vorliegenden Arbeit, die als Dissertation unter der Betreuung von Ignaz Seidl-Hohenveldern an der Universität Köln entstand, geht der Verfasser Peter Hamacher der Frage nach, ob und inwieweit der genannte Grundsatz auch im Bereich des Völkerrechts Geltung beanspruchen kann.

Im ersten Teil der Arbeit befaßt sich der Autor zunächst mit der Problematik des rechtlichen Gehörs in der gutachtlichen Tätigkeit des IGH. Nach einigen einleitenden Aus- 
führungen methodischer Natur werden vorrangig die generellen Schwierigkeiten gerichtlicher Gutachtentätigkeit aufgezeigt, welche sich insbesondere aus deren Unverbindlichkeit für die Parteien ergeben. Die in diesem Rahmen entwickelten Thesen werden sodann auf das Gutachtenverfahren des IGH übertragen, wobei die sich aus der Völkerrechtsordnung ergebenden Besonderheiten deutlich herausgearbeitet sind. Es ergibt sich danach zum einen die Definition des Gerichtsgutachtens als unverbindliche, soweit wie möglich an das streitige Verfahren anzugleichende und auf dem Konsens der Rechtsgenossen beruhende eigenständige Verfahrensart (S. 65), zum anderen die Erkenntnis, daß die Besonderheit der IGH-Gutachten darin liege, die letztgenannte Voraussetzung gerade nicht zu erfüllen. Die Maxime sei in diesem Zusammenhang zwar allgegenwärtig, verliere jedoch die mit ihrem innerstaatlichen Verständnis verbundenen klaren Konturen. Darauffolgend befaßt sich der Autor mit dem Prinzip des rechtlichen Gehörs (des fairen Verfahrens, des "due process of law") als allgemeinem Rechtsgrundsatz, wobei allerdings nicht recht deutlich wird, warum die höchst abstrakten Ausführungen zu den Grundlagen dieser Rechtsfigur nicht an den Anfang der Arbeit gestellt wurden. Nichtsdestoweniger ist es das Verdienst des Verfassers, mit großer Genauigkeit den inhaltlichen und funktionalen Verzweigungen der Maxime nachzuspüren und daneben auch ein Bild von deren historischer Entwicklung zu skizzieren.

Als Ergebnis dieser Uberlegungen wird zunächst eine Definition der Maxime als "je nach Verfahrensziel und Prozeßart variable Gewähr eines Prozeßbetroffenen, durch Äußerungen aller Art auf die Entscheidung Einfluß zu nehmen" gewonnen (S. 119). Die Maxime ruhe dabei auf den Grundlagen der menschlichen Würde und des Selbstbehauptungswillens und sei daher unabdingbarer Maßstab für jedes gerichtliche Handeln. Daraus wird gefolgert, daß der genannte Grundsatz in der beschriebenen Allgemeinheit auch in der von der Gleichordnung der Rechtssubjekte geprägten Völkerrechtsordnung Geltung habe. Des weiteren wird die Frage aufgeworfen, ob und inwieweit "audiatur et altera pars" für einen völkerrechtlich begründeten Individualrechtsschutz nutzbar gemacht werden kann. Anhand einer Reihe universeller bzw. regionaler Konventionen zeigt der Autor, daß der Grundsatz des rechtlichen Gehörs bereits Eingang in die völkerrechtliche Praxis gefunden hat, auch soweit diese dem Individuum Rechtsschutz gewährt. Allerdings wird festgestellt, daß der Grundsatz anders als im innerstaatlichen Recht nicht eine formal-prozessuale Ausformung erfährt, sondern als - formal kaum abgesichertes - materielles Prinzip die einzelnen Verfahren durchzieht.

Ein weiterer Unterabschnitt ist der Tätigkeit der internationalen Verwaltungsgerichte gewidmet, wobei auch hier die Maxime - widerum in einer stärker materiellen als verfahrensrechtlichen Ausprägung - auf das gesamte Verfahren Einfluß habe. Schließlich weist der Autor einen solchen Einfluß auch für solche bilateralen Vereinbarungen der Staaten nach, die individualrechtschützenden Charakter haben.

Am Ende der Arbeit zieht Hamacher aus den zuvor angestellten Uberlegungen eine Reihe von Schlußfolgerungen. So ist er der Meinung, "audiatur et altera pars" sei sowohl als allgemeiner Grundsatz wie auch als individualschützende Norm Inhalt des geltenden Völkerrechts. $\mathrm{Zu}$ einer Formel verdichtet ließe sich also sagen: "Jedermann hat nach 
geltendem Völkerrecht einen Anspruch darauf, daß über seine Interessen gerichtlich nur disponiert werde, wenn für ihn die Gelegenheit bestanden habe, auf das Verfahren Einfluß zu nehmenu (S. 217).

Dabei soll die Maxime nicht nur einen allgemeinen Rechtsgrundsatz im Sinne des Art. $38 \mathrm{Nr} .1$ lit.c. IGH-Statut beinhalten, vielmehr soll sie darüberhinaus auch aus dem Völkergewohnheitsrecht ableitbar sein und dort als eine Art verfahrensrechtlicher Sicherung der Menschenrechte dienen. Es wird sogar erwogen, dem Grundsatz in dieser Hinsicht die Qualität von ius cogens beizulegen.

Zumindest die letztgenannten Schlußfolgerungen erscheinen, so wünschenswert ihre Verwirklichung auch sein mag, unter dem Aspekt entgegenstehender Staatenpraxis angreifbar. Dies verkennt letztlich auch der Autor nicht, wenn er einräumt, daß stets eine Lücke zwischen dem Geltungsanspruch der Maxime und ihrer effektiven Durchsetzung bestehen bleibe.

Das eigentliche Verdienst Hamachers liegt jedoch in dem mit bemerkenswerter Klarheit geführten Nachweis, daß die in den nationalen Rechtsordnungen verankerte Maxime "audiatur et altera pars" auch im Völkerrecht Bedeutung hat. Die Folgerungen Hamachers in Bezug auf deren Geltung für den völkerrechtlichen Individualrechtschutz sind hingegen wohl eher als Hoffnung de lege ferenda denn als bereits allseits geübte Staatenpraxis zu bezeichnen.

Michael Seitz

\section{Walter Rudolf,}

\section{Wandel des Staatsbegriffs im Völkerrecht?}

Schriftenreihe des Walter-Schücking-Kollegs, Bonn, Europa Union Verlag, 1986, 48 S., DM 9,-

Die Schrift gibt einen um Fußnoten bereicherten Vortrag wieder, den Walter Rudolf im Rahmen des Walter-Schücking-Kollegs im Februar 1983 an der Universität Kiel gehalten hat. In ihm zeichnet Rudolf so präzis und umfangreich, wie es die Vortragsform nur zuläßt, die Entwicklung nach, die die Souveränität der Staaten seit der Entstehung des modernen Völkerrechts genommen hat. Er kommt dabei zu dem Ergebnis, daß selbst so tiefgreifende gesellschaftspolitische Veränderungen wie z. B. der Dekolonisierungsprozeß nach 1945 keine strukturellen Veränderungen der Völkerrechtsordnung bewirkten. Solange diese aber als Koordinationsrecht, d. h. als Recht, das keinen Staat gegen seinen Willen dem Willen anderer Staaten unterwirft, bestehen bleibt, werde sich auch der Staatenbegriff nicht wandeln. Tendenzen, diese Struktur zu ändern, beurteilt Rudolf" de lege lata" skeptisch, ob eine solche Entwicklung "de lege ferenda " wünschenswert wäre, dazu enthält er sich jeder Wertung.

Andrea Franke 